\title{
Linked partition ideals and the Alladi-Schur theorem
}

\author{
George E. Andrews, Shane Chern, and Zhitai Li
}

\begin{abstract}
Let $\mathscr{S}$ denote the set of integer partitions into parts that differ by at least 3 , with the added constraint that no two consecutive multiples of 3 occur as parts. We derive trivariate generating functions of Andrews-Gordon type for partitions in $\mathscr{S}$ with both the number of parts and the number of even parts counted. In particular, we provide an analytic counterpart of Andrews' recent refinement of the Alladi-Schur theorem.
\end{abstract}

Keywords. Linked partition ideals, Alladi-Schur theorem, Andrews-Gordon type series, generating function.

2010MSC. 11P84, 05A17.

\section{Introduction}

The following result was proved by Schur [19] in 1926.

Theorem S. Let $A(n)$ denote the number of partitions of $n$ into parts congruent to \pm 1 modulo 6 . Let $B(n)$ denote the number of partitions of $n$ into distinct nonmultiples of 3 . Let $D(n)$ denote the number of partitions of $n$ of the form $\mu_{1}+\mu_{2}+\cdots+\mu_{s}$ where $\mu_{i}-\mu_{i+1} \geq 3$ with strict inequality if $3 \mid \mu_{i}$. Then

$$
A(n)=B(n)=D(n) .
$$

Alladi later made an observation (cf. [7, p. 46, (1.3)]).

Theorem AS. If we define $C(n)$ to be the number of partitions of $n$ into odd parts with none appearing more than twice, then

$$
C(n)=D(n) .
$$

On the other hand, Gleißberg [14] had a refinement of Theorem S.

Theorem G. Let $B(m, n)$ denote the number of partitions of $n$ into $m$ distinct nonmultiples of 3 . Let $D(m, n)$ denote the number of partitions of $n$ enumerated by $D(n)$ such that the total number of parts plus the number of multiples of 3 among the parts equals $m$. Then

$$
B(m, n)=D(m, n) .
$$

Motivated by Gleißberg's result, Andrews $[8,9]$ also refined Theorem AS in the same manner.

Theorem A. Let $C(m, n)$ denote the number of partitions of $n$ into $m$ odd parts with none appearing more than twice. Let $D^{\prime}(m, n)$ denote the number of partitions of $n$ enumerated by $D(n)$ such that the total number of parts plus the number of even parts equals $m$. Then

$$
C(m, n)=D^{\prime}(m, n)
$$


A surprising point regarding Theorem $\mathrm{A}$ is that partitions enumerated by $D(n)$ are controlled by a difference condition of size 3 (so in terms of linked partition ideals, which will be explained in the next section, the modulus is 3 ), and therefore less attention was paid to even parts in the previous studies.

Recall also that an Andrews-Gordon type series is of the form

$$
\sum_{n_{1}, \ldots, n_{r} \geq 0} \frac{(-1)^{L_{1}\left(n_{1}, \ldots, n_{r}\right)} q^{Q\left(n_{1}, \ldots, n_{r}\right)+L_{2}\left(n_{1}, \ldots, n_{r}\right)}}{\left(q^{A_{1}} ; q^{A_{1}}\right)_{n_{1}} \cdots\left(q^{A_{r}} ; q^{A_{r}}\right)_{n_{r}}},
$$

in which $L_{1}$ and $L_{2}$ are linear forms and $Q$ is a quadratic form in $n_{1}, \ldots, n_{r}$, and the $q$-Pochhammer symbol is defined for $n \in \mathbb{N} \cup\{\infty\}$,

$$
(A ; q)_{n}:=\prod_{k=0}^{n-1}\left(1-A q^{k}\right)
$$

This terminology comes from the multiple-folder generalization of the RogersRamanujan identities due to Andrews [3] and Gordon [15]: for $1 \leq i \leq k$ and $k \geq 2$,

$$
\prod_{\substack{n \geq 1 \\ n \neq 0, \pm i(\bmod 2 k+1)}} \frac{1}{1-q^{n}}=\sum_{n_{1}, \ldots, n_{k-1} \geq 0} \frac{q^{N_{1}^{2}+N_{2}^{2}+\cdots N_{k-1}^{2}+N_{i}+N_{i+1}+\cdots+N_{k-1}}}{(q ; q)_{n_{1}}(q ; q)_{n_{2}} \cdots(q ; q)_{n_{k-1}}}
$$

where $N_{j}=n_{j}+n_{j+1}+\cdots+n_{k-1}$.

Let $\mathscr{S}$ denote the set of partitions enumerated by $D(n)$ for all nonnegative $n$.

Further, for any partition $\lambda$, we denote by $|\lambda|$ the sum of all parts in $\lambda$, by $\sharp(\lambda)$ the number of parts in $\lambda$, and by $\sharp_{a, M}(\lambda)$ the number of parts in $\lambda$ that are congruent to a modulo $M$.

Many discoveries have been made on the generating function of Andrews-Gordon type for the partition set $\mathscr{S}$. In particular, Andrews, Bringmann and Mahlburg [10] proved that

$$
\sum_{\lambda \in \mathscr{S}} x^{\sharp(\lambda)} q^{|\lambda|}=\sum_{m, n \geq 0} \frac{(-1)^{n} q^{(m+3 n)^{2}+\frac{m(m-1)}{2} x^{m+2 n}}}{(q ; q)_{m}\left(q^{6} ; q^{6}\right)_{n}},
$$

Kurşungöz [17] proved that

$$
\begin{aligned}
& \sum_{\lambda \in \mathscr{S}} x^{\sharp(\lambda)} q^{|\lambda|} \\
& =\sum_{\substack{n_{1} \geq 0 \\
n_{21}, n_{22} \geq 0}} \frac{q^{2 n_{1}^{2}-n_{1}+6 n_{21}^{2}-n_{21}+6 n_{22}^{2}+n_{22}+6 n_{1}\left(n_{21}+n_{22}\right)+12 n_{21} n_{22}} x^{n_{1}+2 n_{21}+2 n_{22}}}{(q ; q)_{n_{1}}\left(q^{6} ; q^{6}\right)_{n_{21}}\left(q^{6} ; q^{6}\right)_{n_{22}}},
\end{aligned}
$$

and Alladi and Gordon [1] proved that

$$
\begin{aligned}
& \sum_{\lambda \in \mathscr{S}} x^{\sharp(\lambda)} y^{\sharp 0,3(\lambda)} q^{|\lambda|} \\
& \quad=\sum_{n_{1}, n_{2}, n_{3} \geq 0} \frac{q^{3\left(\begin{array}{c}
n_{1} \\
2
\end{array}\right)+3\left(\begin{array}{c}
n_{2} \\
2
\end{array}\right)+6\left(\begin{array}{c}
n_{3} \\
2
\end{array}\right)+3 n_{1} n_{2}+3 n_{2} n_{3}+3 n_{3} n_{1}+n_{1}+2 n_{2}+3 n_{3}} x^{n_{1}+n_{2}+n_{3}} y^{n_{3}}}{\left(q^{3} ; q^{3}\right)_{n_{1}}\left(q^{3} ; q^{3}\right)_{n_{2}}\left(q^{3} ; q^{3}\right)_{n_{3}}} .
\end{aligned}
$$


Recently, using the framework of linked partition ideals, Chern [12] reproduced (1.2) and further proved that if $\mathscr{S}_{S}$ denotes the set of partitions in $\mathscr{S}$ whose smallest part is not in the set $S$ of positive integers, then

$$
\begin{aligned}
& \sum_{\lambda \in \mathscr{S}_{\{1\}}} x^{\sharp(\lambda)} y^{\sharp 0,3}(\lambda) q^{|\lambda|} \\
& \quad=\sum_{n_{1}, n_{2}, n_{3} \geq 0} \frac{q^{3\left(\begin{array}{c}
n_{1} \\
2
\end{array}\right)+3\left(\begin{array}{c}
n_{2} \\
2
\end{array}\right)+6\left(\begin{array}{c}
n_{3} \\
2
\end{array}\right)+3 n_{1} n_{2}+3 n_{2} n_{3}+3 n_{3} n_{1}+4 n_{1}+2 n_{2}+3 n_{3}} x^{n_{1}+n_{2}+n_{3}} y^{n_{3}}}{\left(q^{3} ; q^{3}\right)_{n_{1}}\left(q^{3} ; q^{3}\right)_{n_{2}}\left(q^{3} ; q^{3}\right)_{n_{3}}}
\end{aligned}
$$

and

$$
\begin{aligned}
& \sum_{\lambda \in \mathscr{S}_{\{1,2,3\}}} x^{\sharp(\lambda)} y^{\sharp 0,3}(\lambda) q^{|\lambda|} \\
& =\sum_{n_{1}, n_{2}, n_{3} \geq 0} \frac{q^{3\left(\begin{array}{c}
n_{1} \\
2
\end{array}\right)+3\left(\begin{array}{c}
n_{2} \\
2
\end{array}\right)+6\left(\begin{array}{c}
n_{3} \\
2
\end{array}\right)+3 n_{1} n_{2}+3 n_{2} n_{3}+3 n_{3} n_{1}+4 n_{1}+5 n_{2}+6 n_{3}} x^{n_{1}+n_{2}+n_{3}} y^{n_{3}}}{\left(q^{3} ; q^{3}\right) n_{1}\left(q^{3} ; q^{3}\right) n_{2}\left(q^{3} ; q^{3}\right) n_{3}} .
\end{aligned}
$$

Notice that (1.2) yields an analytic counterpart of Theorem $\mathrm{G}$ by setting $y=x$ :

$$
\begin{aligned}
\left(-x q ; q^{3}\right)_{\infty}\left(-x q^{2} ; q^{3}\right)_{\infty} \\
\quad=\sum_{n_{1}, n_{2}, n_{3} \geq 0} \frac{q^{3\left(\begin{array}{c}
n_{1} \\
2
\end{array}\right)+3\left(\begin{array}{c}
n_{2} \\
2
\end{array}\right)+6\left(\begin{array}{c}
n_{3} \\
2
\end{array}\right)+3 n_{1} n_{2}+3 n_{2} n_{3}+3 n_{3} n_{1}+n_{1}+2 n_{2}+3 n_{3}} x^{n_{1}+n_{2}+2 n_{3}}}{\left(q^{3} ; q^{3}\right)_{n_{1}}\left(q^{3} ; q^{3}\right)_{n_{2}}\left(q^{3} ; q^{3}\right)_{n_{3}}} .
\end{aligned}
$$

With Theorem A in mind, our object is the following result.

Theorem 1.1. We have

$$
\begin{aligned}
& \sum_{\lambda \in \mathscr{S}} x^{\sharp(\lambda)} y^{\sharp 0,2}(\lambda) q^{|\lambda|}=\sum_{n_{1}, n_{2}, n_{3} \geq 0} \frac{(-1)^{n_{3}} x^{n_{1}+n_{2}+2 n_{3}} y^{n_{2}+n_{3}}}{\left(q^{2} ; q^{2}\right)_{n_{1}}\left(q^{2} ; q^{2}\right)_{n_{2}}\left(q^{6} ; q^{6}\right)_{n_{3}}} \\
& \times q^{4\left(\begin{array}{c}
n_{1} \\
2
\end{array}\right)+4\left(\begin{array}{c}
n_{2} \\
2
\end{array}\right)+18\left(\begin{array}{c}
n_{3} \\
2
\end{array}\right)+2 n_{1} n_{2}+6 n_{2} n_{3}+6 n_{3} n_{1}+n_{1}+2 n_{2}+9 n_{3},} \\
& \sum_{\lambda \in \mathscr{S}_{\{1\}}} x^{\sharp(\lambda)} y^{\sharp 0,2}(\lambda) q^{|\lambda|}=\sum_{n_{1}, n_{2}, n_{3} \geq 0} \frac{(-1)^{n_{3}} x^{n_{1}+n_{2}+2 n_{3}} y^{n_{2}+n_{3}}}{\left(q^{2} ; q^{2}\right)_{n_{1}}\left(q^{2} ; q^{2}\right)_{n_{2}}\left(q^{6} ; q^{6}\right)_{n_{3}}} \\
& \times q^{4\left(\begin{array}{c}
n_{1} \\
2
\end{array}\right)+4\left(\begin{array}{c}
n_{2} \\
2
\end{array}\right)+18\left(\begin{array}{c}
n_{3} \\
2
\end{array}\right)+2 n_{1} n_{2}+6 n_{2} n_{3}+6 n_{3} n_{1}+3 n_{1}+2 n_{2}+9 n_{3},} \\
& \sum_{\lambda \in \mathscr{S}_{\{1,2,3\}}} x^{\sharp(\lambda)} y^{\sharp 0,2(\lambda)} q^{|\lambda|}=\sum_{n_{1}, n_{2}, n_{3} \geq 0} \frac{(-1)^{n_{3}} x^{n_{1}+n_{2}+2 n_{3}} y^{n_{2}+n_{3}}}{\left(q^{2} ; q^{2}\right)_{n_{1}}\left(q^{2} ; q^{2}\right)_{n_{2}}\left(q^{6} ; q^{6}\right)_{n_{3}}} \\
& \times q^{4\left(\begin{array}{c}
n_{1} \\
2
\end{array}\right)+4\left(\begin{array}{c}
n_{2} \\
2
\end{array}\right)+18\left(\begin{array}{c}
n_{3} \\
2
\end{array}\right)+2 n_{1} n_{2}+6 n_{2} n_{3}+6 n_{3} n_{1}+5 n_{1}+4 n_{2}+15 n_{3} .}
\end{aligned}
$$

In particular, (1.6) implies a corollary as follows. 
Corollary 1.2. Theorem A is true. Also, it has an analytic counterpart

$$
\begin{aligned}
\prod_{n \geq 0}\left(1+x q^{2 n+1}+x^{2} q^{4 n+2}\right) & =\sum_{n_{1}, n_{2}, n_{3} \geq 0} \frac{(-1)^{n_{3}} x^{n_{1}+2 n_{2}+3 n_{3}}}{\left(q^{2} ; q^{2}\right)_{n_{1}}\left(q^{2} ; q^{2}\right)_{n_{2}}\left(q^{6} ; q^{6}\right)_{n_{3}}} \\
& \times q^{4\left(\begin{array}{c}
n_{1} \\
2
\end{array}\right)+4\left(\begin{array}{c}
n_{2} \\
2
\end{array}\right)+18\left(\begin{array}{c}
n_{3} \\
2
\end{array}\right)+2 n_{1} n_{2}+6 n_{2} n_{3}+6 n_{3} n_{1}+n_{1}+2 n_{2}+9 n_{3}} .
\end{aligned}
$$

Our paper is organized as follows. In Section 2, we give a brief review of linked partition ideals and use this framework to construct a matrix equation for generating functions related to the partition set $\mathscr{S}$. Then in Sections 3-6, we present a proof of (1.6). The basic idea comes from the work of Chern and Li [13]. For (1.7) and (1.8), we may proceed in a similar way so the details will be omitted. Next, we prove Corollary 1.2 in Section 7. Here, we provide two proofs - one is

$q$-hypergeometric and the other is computer-assisted. Finally, we close this paper with a conclusion in Section 8.

\section{Linked partition ideals and a matrix equation}

2.1. Span one linked partition ideals. The concept of linked partition ideals was introduced by Andrews $[2,4,5]$ in the 1970s for a general theory of partition identities. We refer the interested reader to [6, Chapter 8] for a detailed account. Recently, Chern and Li [13] and Chern [11] revisited a special type of linked partition ideals, called span one linked partition ideals, in their study of Andrews-Gordon type series for several partition sets.

Definition 2.1. Assume that we are given

- a finite set $\Pi=\left\{\pi_{1}, \pi_{2}, \ldots, \pi_{K}\right\}$ of integer partitions with $\pi_{1}=\emptyset$, the empty partition,

- a map of linking sets, $\mathcal{L}: \Pi \rightarrow P(\Pi)$, the power set of $\Pi$, with especially, $\mathcal{L}\left(\pi_{1}\right)=\mathcal{L}(\emptyset)=\Pi$ and $\pi_{1}=\emptyset \in \mathcal{L}\left(\pi_{k}\right)$ for any $1 \leq k \leq K$,

- and a positive integer $T$, called the modulus, which is greater than or equal to the largest part among all partitions in $\Pi$.

We say a span one linked partition ideal $\mathscr{I}=\mathscr{I}(\langle\Pi, \mathcal{L}\rangle, T)$ is the collection of all partitions of the form

$$
\begin{aligned}
\lambda & =\phi^{0}\left(\lambda_{0}\right) \oplus \phi^{T}\left(\lambda_{1}\right) \oplus \cdots \oplus \phi^{N T}\left(\lambda_{N}\right) \oplus \phi^{(N+1) T}\left(\pi_{1}\right) \oplus \phi^{(N+2) T}\left(\pi_{1}\right) \oplus \cdots \\
& =\phi^{0}\left(\lambda_{0}\right) \oplus \phi^{T}\left(\lambda_{1}\right) \oplus \cdots \oplus \phi^{N T}\left(\lambda_{N}\right),
\end{aligned}
$$

where $\lambda_{i} \in \mathcal{L}\left(\lambda_{i-1}\right)$ for each $i$ and $\lambda_{N}$ is not the empty partition. We also include in $\mathscr{I}$ the empty partition, which corresponds to $\phi^{0}\left(\pi_{1}\right) \oplus \phi^{T}\left(\pi_{1}\right) \oplus \cdots$. Here for any two partitions $\mu$ and $\nu, \mu \oplus \nu$ gives a partition by collecting all parts in $\mu$ and $\nu$, and $\phi^{m}(\mu)$ gives a partition by adding $m$ to each part of $\mu$.

It was shown in [12] that the partition set $\mathscr{S}$ in Schur's theorem is the span one linked partition ideal $\mathscr{I}\left(\left\langle\Pi^{\prime}, \mathcal{L}^{\prime}\right\rangle, 3\right)$, where $\Pi^{\prime}=\left\{\varpi_{1}=\emptyset, \varpi_{2}=(1), \varpi_{3}=(2), \varpi_{4}=\right.$ 
(3)\} and

$$
\left\{\begin{array}{l}
\mathcal{L}^{\prime}\left(\varpi_{1}\right)=\left\{\varpi_{1}, \varpi_{2}, \varpi_{3}, \varpi_{4}\right\}, \\
\mathcal{L}^{\prime}\left(\varpi_{2}\right)=\left\{\varpi_{1}, \varpi_{2}, \varpi_{3}, \varpi_{4}\right\}, \\
\mathcal{L}^{\prime}\left(\varpi_{3}\right)=\left\{\varpi_{1}, \varpi_{3}, \varpi_{4}\right\}, \\
\mathcal{L}^{\prime}\left(\varpi_{4}\right)=\left\{\varpi_{1}\right\} .
\end{array}\right.
$$

However, this equivalence is not sufficient when even parts are taken into consideration. Therefore, our first goal is the following refinement.

Lemma 2.1. $\mathscr{S}$ is also the span one linked partition ideal $\mathscr{I}(\langle\Pi, \mathcal{L}\rangle, 6)$, where $\Pi=\left\{\pi_{1}=\emptyset, \pi_{2}=(1), \pi_{3}=(2), \pi_{4}=(3), \pi_{5}=(4), \pi_{6}=(1+4), \pi_{7}=(5), \pi_{8}=\right.$ $\left.(1+5), \pi_{9}=(2+5), \pi_{10}=(6), \pi_{11}=(1+6), \pi_{12}=(2+6)\right\}$ and

$$
\left\{\begin{aligned}
\mathcal{L}\left(\pi_{1}\right)=\mathcal{L}\left(\pi_{2}\right)=\cdots=\mathcal{L}\left(\pi_{6}\right) & =\left\{\pi_{1}, \pi_{2}, \ldots, \pi_{12}\right\} \\
\mathcal{L}\left(\pi_{7}\right)=\mathcal{L}\left(\pi_{8}\right)=\mathcal{L}\left(\pi_{9}\right) & =\left\{\pi_{1}, \pi_{3}, \pi_{4}, \pi_{5}, \pi_{7}, \pi_{9}, \pi_{10}, \pi_{12}\right\} \\
\mathcal{L}\left(\pi_{10}\right)=\mathcal{L}\left(\pi_{11}\right)=\mathcal{L}\left(\pi_{12}\right) & =\left\{\pi_{1}, \pi_{5}, \pi_{7}, \pi_{10}\right\}
\end{aligned}\right.
$$

Proof. We decompose each partition in $\mathscr{S}$ into blocks $B_{0}, B_{1}, \ldots$ such that all parts between $6 i+1$ and $6 i+6$ fall into block $B_{i}$. By the definition of $\mathscr{S}$, we find that if we apply the operator $\phi^{-6 i}$ to the block $B_{i}$, then it is among $\Pi$. If $\phi^{-6 i}\left(B_{i}\right)$ is any of $\left\{\pi_{1}, \pi_{2}, \ldots, \pi_{6}\right\}$, then the largest part in block $B_{i}$ is at most $6 i+4$, and therefore $\phi^{-6(i+1)}\left(B_{i+1}\right)$ can be any among $\Pi$. If $\phi^{-6 i}\left(B_{i}\right)$ is any of $\left\{\pi_{7}, \pi_{8}, \pi_{9}\right\}$, then the largest part in block $B_{i}$ is $6 i+5$, and therefore the smallest part in block $B_{i+1}$ is at least $6(i+1)+2$, that is $\phi^{-6(i+1)}\left(B_{i+1}\right) \notin\left\{\pi_{2}, \pi_{6}, \pi_{8}, \pi_{11}\right\}$. If $\phi^{-6 i}\left(B_{i}\right)$ is any of $\left\{\pi_{10}, \pi_{11}, \pi_{12}\right\}$, then the largest part in block $B_{i}$ is $6 i+6$, and therefore the smallest part in block $B_{i+1}$ is at least $6(i+1)+4$, that is $\phi^{-6(i+1)}\left(B_{i+1}\right) \notin$ $\left\{\pi_{2}, \pi_{3}, \pi_{4}, \pi_{6}, \pi_{8}, \pi_{9}, \pi_{11}, \pi_{12}\right\}$. Hence, all partitions in $\mathscr{S}$ are also in $\mathscr{I}(\langle\Pi, \mathcal{L}\rangle, 6)$. Conversely, it is straightforward to verify that all partitions in $\mathscr{I}(\langle\Pi, \mathcal{L}\rangle, 6)$ satisfy the difference conditions defined for $D(n)$, and are thus in $\mathscr{S}$.

2.2. A matrix equation. For any partition $\lambda \in \mathscr{S}=\mathscr{I}(\langle\Pi, \mathcal{L}\rangle, 6)$, we always decompose it as in (2.1). For each $1 \leq k \leq 12$, we write

$$
G_{k}(x):=\sum_{\substack{\lambda \in \mathscr{S} \\ \lambda_{0}=\pi_{k}}} x^{\sharp(\lambda)} y^{\sharp 0,2(\lambda)} q^{|\lambda|},
$$

that is, $G_{k}(x)$ is the generating function for partitions whose first decomposed block is $\pi_{k}$. By the definition of span one linked partition ideals, we have

$$
G_{k}(x)=x^{\sharp\left(\pi_{k}\right)} y^{\sharp 0,2}\left(\pi_{k}\right) q^{\left|\pi_{k}\right|} \sum_{j: \pi_{j} \in \mathcal{L}\left(\pi_{k}\right)} G_{j}\left(x q^{6}\right) .
$$

Therefore,

$$
\left(\begin{array}{c}
G_{1}(x) \\
G_{2}(x) \\
\vdots \\
G_{12}(x)
\end{array}\right)=\mathscr{W}(x) \cdot \mathscr{A} \cdot\left(\begin{array}{c}
G_{1}\left(x q^{6}\right) \\
G_{2}\left(x q^{6}\right) \\
\vdots \\
G_{12}\left(x q^{6}\right)
\end{array}\right)
$$

where

$$
\mathscr{W}(x)=\operatorname{diag}\left(1, x q, x y q^{2}, x q^{3}, x y q^{4}, x^{2} y q^{5}, x q^{5}, x^{2} q^{6}, x^{2} y q^{7}, x y q^{6}, x^{2} y q^{7}, x^{2} y^{2} q^{8}\right)
$$


and

$$
\mathscr{A}=\left(\begin{array}{llllllllllll}
1 & 1 & 1 & 1 & 1 & 1 & 1 & 1 & 1 & 1 & 1 & 1 \\
1 & 1 & 1 & 1 & 1 & 1 & 1 & 1 & 1 & 1 & 1 & 1 \\
1 & 1 & 1 & 1 & 1 & 1 & 1 & 1 & 1 & 1 & 1 & 1 \\
1 & 1 & 1 & 1 & 1 & 1 & 1 & 1 & 1 & 1 & 1 & 1 \\
1 & 1 & 1 & 1 & 1 & 1 & 1 & 1 & 1 & 1 & 1 & 1 \\
1 & 1 & 1 & 1 & 1 & 1 & 1 & 1 & 1 & 1 & 1 & 1 \\
1 & 0 & 1 & 1 & 1 & 0 & 1 & 0 & 1 & 1 & 0 & 1 \\
1 & 0 & 1 & 1 & 1 & 0 & 1 & 0 & 1 & 1 & 0 & 1 \\
1 & 0 & 1 & 1 & 1 & 0 & 1 & 0 & 1 & 1 & 0 & 1 \\
1 & 0 & 0 & 0 & 1 & 0 & 1 & 0 & 0 & 1 & 0 & 0 \\
1 & 0 & 0 & 0 & 1 & 0 & 1 & 0 & 0 & 1 & 0 & 0 \\
1 & 0 & 0 & 0 & 1 & 0 & 1 & 0 & 0 & 1 & 0 & 0
\end{array}\right)
$$

We further write

$$
\left(\begin{array}{c}
F_{1}(x) \\
F_{2}(x) \\
\vdots \\
F_{12}(x)
\end{array}\right)=\mathscr{A} \cdot\left(\begin{array}{c}
G_{1}(x) \\
G_{2}(x) \\
\vdots \\
G_{12}(x)
\end{array}\right)
$$

Then

$$
\left(\begin{array}{c}
F_{1}(x) \\
F_{2}(x) \\
\vdots \\
F_{12}(x)
\end{array}\right)=\mathscr{A} \cdot \mathscr{W}(x) \cdot \mathscr{A} \cdot\left(\begin{array}{c}
G_{1}\left(x q^{6}\right) \\
G_{2}\left(x q^{6}\right) \\
\vdots \\
G_{12}\left(x q^{6}\right)
\end{array}\right)
$$

and therefore,

$$
\left(\begin{array}{c}
F_{1}(x) \\
F_{2}(x) \\
\vdots \\
F_{12}(x)
\end{array}\right)=\mathscr{A} \cdot \mathscr{W}(x) .\left(\begin{array}{c}
F_{1}\left(x q^{6}\right) \\
F_{2}\left(x q^{6}\right) \\
\vdots \\
F_{12}\left(x q^{6}\right)
\end{array}\right) .
$$

Also, (2.4) implies that

$$
\begin{array}{r}
F_{1}(x)=F_{2}(x)=\cdots=F_{6}(x)=: A_{1}(x), \\
F_{7}(x)=F_{8}(x)=F_{9}(x)=: A_{2}(x), \\
F_{10}(x)=F_{11}(x)=F_{12}(x)=: A_{3}(x) .
\end{array}
$$

We may rewrite (2.5) as

$$
\left(\begin{array}{l}
A_{1}(x) \\
A_{2}(x) \\
A_{3}(x)
\end{array}\right)=\mathscr{M} \cdot\left(\begin{array}{c}
A_{1}\left(x q^{6}\right) \\
A_{2}\left(x q^{6}\right) \\
A_{3}\left(x q^{6}\right)
\end{array}\right)
$$

where

$$
\mathscr{M}=\left(\begin{array}{ccc}
1+x q+x y q^{2}+x q^{3}+x y q^{4}+x^{2} y q^{5} & x q^{5}+x^{2} q^{6}+x^{2} y q^{7} & x y q^{6}+x^{2} y q^{7}+x^{2} y^{2} q^{8} \\
1+x y q^{2}+x q^{3}+x y q^{4} & x q^{5}+x^{2} y q^{7} & x y q^{6}+x^{2} y^{2} q^{8} \\
1+x y q^{4} & x q^{5} & x y q^{6}
\end{array}\right) .
$$


Finally, we observe that

$$
\begin{aligned}
& \sum_{\lambda \in \mathscr{S}} x^{\sharp(\lambda)} y^{\sharp 0,2(\lambda)} q^{|\lambda|}=\sum_{k \in\{1,2,3, \ldots, 12\}} G_{k}(x) \\
& =A_{1}(x) \text {, } \\
& \sum_{\lambda \in \mathscr{S}_{\{1\}}} x^{\sharp(\lambda)} y^{\sharp 0,2}(\lambda) q^{|\lambda|}=\sum_{k \in\{1,3,4,5,7,9,10,12\}} G_{k}(x) \\
& =A_{2}(x) \text {, } \\
& \sum_{\lambda \in \mathscr{S}_{\{1,2,3\}}} x^{\sharp(\lambda)} y^{\sharp 0,2}(\lambda) q^{|\lambda|}=\sum_{k \in\{1,5,7,10\}} G_{k}(x) \\
& =A_{3}(x) \text {. }
\end{aligned}
$$

\section{A $q$-difference equation}

In Sections 3-6, we present a proof of (1.6), following the idea in [13]. We will omit the proofs of (1.7) and (1.8). However, all related Mathematica codes were uploaded at the following url:

\section{https://github.com/shanechern/Alladi-Schur}

In order to reduce matrix equations such as (2.9) to $q$-difference equations of $A_{1}(x), A_{2}(x)$ and $A_{3}(x)$, an algorithm was presented in [13, Section 3], following the idea in [6, Lemma 8.10]. So our first result is as follows.

Theorem 3.1. Let $A_{1}(x)$ be as in (2.9). Then

$$
\begin{aligned}
0=[1 & \left.+x\left(q^{7}+y q^{8}\right)\right] A_{1}(x) \\
- & {\left[1+x\left(q+q^{3}+q^{5}+q^{7}+y q^{2}+y q^{4}+y q^{6}+y q^{8}\right)\right.} \\
& +x^{2}\left(q^{6}+q^{8}+q^{10}+y q^{5}+2 y q^{7}+2 y q^{9}+2 y q^{11}+y q^{13}+y^{2} q^{8}+y^{2} q^{10}+y^{2} q^{12}\right) \\
& \left.+x^{3}\left(y q^{12}+y q^{14}+y^{2} q^{13}+y^{2} q^{15}\right)\right] A_{1}\left(x q^{6}\right) \\
+ & {\left[x^{2} y q^{15}+x^{3}\left(-q^{21}+y q^{16}+y^{2} q^{17}-y^{3} q^{24}\right)\right.} \\
& +x^{4}\left(-q^{22}-y q^{23}+y^{2} q^{30}-y^{3} q^{25}-y^{4} q^{26}\right) \\
& \left.+x^{5}\left(y^{2} q^{31}+y^{3} q^{32}\right)\right] A_{1}\left(x q^{12}\right) .
\end{aligned}
$$

Proof. This result follows from an implementation of the algorithm in [13, Section 3]. Detailed calculations can be found in the Mathematica notebook AS-1.nb uploaded at the url listed at the beginning of Section 3.

Let

$$
A_{1}(x)=\sum_{M \geq 0} a(M) x^{M}
$$

Our next task is to deduce a recurrence for the coefficients $a(M)$. 
Corollary 3.2. For any $M \geq 0$,

$$
\begin{aligned}
0= & q^{12 M}\left(y^{2} q^{31}+y^{3} q^{32}\right) a(M) \\
& +q^{12(M+1)}\left(-q^{22}-y q^{23}+y^{2} q^{30}-y^{3} q^{25}-y^{4} q^{26}\right) a(M+1) \\
& +\left[-q^{6(M+2)}\left(y q^{12}+y q^{14}+y^{2} q^{13}+y^{2} q^{15}\right)\right. \\
& \left.+q^{12(M+2)}\left(-q^{21}+y q^{16}+y^{2} q^{17}-y^{3} q^{24}\right)\right] a(M+2) \\
+ & {\left[-q^{6(M+3)}\left(q^{6}+q^{8}+q^{10}+y q^{5}+2 y q^{7}+2 y q^{9}+2 y q^{11}+y q^{13}+y^{2} q^{8}+y^{2} q^{10}+y^{2} q^{12}\right)\right.} \\
& \left.+q^{12(M+3)} y q^{15}\right] a(M+3) \\
+ & {\left[\left(q^{7}+y q^{8}\right)-q^{6(M+4)}\left(q+q^{3}+q^{5}+q^{7}+y q^{2}+y q^{4}+y q^{6}+y q^{8}\right)\right] a(M+4) } \\
& +\left[1-q^{6(M+5)}\right] a(M+5) .
\end{aligned}
$$

Proof. For each $M \geq 0$, we equate the coefficients of $x^{M+5}$ on both sides of the $q$-difference equation in Theorem 3.1, and thus arrive at the desired recurrence.

\section{Guessing the Andrews-Gordon type series}

Notice that if a series $A(x) \in \mathbb{C}[[y]][[q]][[x]]$ satisfies the $q$-difference equation in Theorem 3.1, then it is uniquely determined by $A(0)$. Recall also that, by (2.10), $A_{1}(0)=1$. Therefore, we can compute the first several terms of $a(M)$ by the $q$-difference equation of $A_{1}(x)$ :

$$
\begin{aligned}
& a(0)=1 \\
& a(1)=\frac{q(1+y q)}{1-q^{2}}, \\
& a(2)=\frac{q^{5}\left(q-q^{7}+y+y q^{2}-y q^{4}-y q^{10}+y^{2} q^{3}-y^{2} q^{9}\right)}{\left(1-q^{2}\right)\left(1-q^{4}\right)\left(1-q^{6}\right)} \\
& a(3)=\frac{q^{12}(1+y q)\left(q^{3}+y+y q^{2}-y q^{4}+y q^{8}+y^{2} q^{5}\right)}{\left(1-q^{2}\right)\left(1-q^{4}\right)\left(1-q^{6}\right)} \\
& a(4)=\frac{(* \cdots *)}{\left(1-q^{2}\right)\left(1-q^{4}\right)\left(1-q^{6}\right)\left(1-q^{8}\right)\left(1-q^{12}\right)} .
\end{aligned}
$$

To guess a suitable candidate of a Andrews-Gordon type series for $A_{1}(x)$, we require a process of brute force. From $a(1)$, it is natural to expect summations of the form:

$$
\sum_{n_{1} \geq 0} \frac{q^{?} x^{n_{1}}}{\left(q^{2} ; q^{2}\right)_{n_{1}}} \quad \text { and } \quad \sum_{n_{2} \geq 0} \frac{q^{?} x^{n_{2}} y^{n_{2}}}{\left(q^{2} ; q^{2}\right)_{n_{2}}} .
$$

From $a(2)$, it is also highly possible that an extra summation is needed:

$$
\sum_{n_{3} \geq 0} \frac{(-1)^{?} q^{?} x^{2 n_{3}} y^{n_{2}}}{\left(q^{6} ; q^{6}\right)_{n_{3}}}
$$

Therefore, we may first try the candidate of a triple summation:

$$
\begin{aligned}
& \sum_{n_{1}, n_{2}, n_{3} \geq 0} \frac{(-1)^{n_{3}} x^{n_{1}+n_{2}+2 n_{3}} y^{n_{2}+n_{3}}}{\left(q^{2} ; q^{2}\right)_{n_{1}}\left(q^{2} ; q^{2}\right)_{n_{2}}\left(q^{6} ; q^{6}\right)_{n_{3}}} \\
& \times q^{\alpha_{11}\left(\begin{array}{c}
n_{1} \\
2
\end{array}\right)+\alpha_{22}\left(\begin{array}{c}
n_{2} \\
2
\end{array}\right)+\alpha_{33}\left(\begin{array}{c}
n_{3} \\
2
\end{array}\right)+\alpha_{12} n_{1} n_{2}+\alpha_{23} n_{2} n_{3}+\alpha_{31} n_{3} n_{1}+\beta_{1} n_{1}+\beta_{2} n_{2}+\beta_{3} n_{3} .}
\end{aligned}
$$


From $a(1)$, we may compute that $\beta_{1}=1$ and $\beta_{2}=2$. From $a(2)$, we may further compute that $\alpha_{11}=4$ and $\alpha_{22}=4$, and single out candidates of $\alpha_{12}=2$ and $\beta_{3}=9$. Continuing this process, we finally arrive at the right-hand side of (1.6).

\section{Another recurrence}

Let

$$
\begin{aligned}
\sum_{M \geq 0} \tilde{a}(M) x^{M} & =\sum_{n_{1}, n_{2}, n_{3} \geq 0} \frac{(-1)^{n_{3}} x^{n_{1}+n_{2}+2 n_{3}} y^{n_{2}+n_{3}}}{\left(q^{2} ; q^{2}\right)_{n_{1}}\left(q^{2} ; q^{2}\right)_{n_{2}}\left(q^{6} ; q^{6}\right)_{n_{3}}} \\
& \times q^{4\left(\begin{array}{c}
n_{1} \\
2
\end{array}\right)+4\left(\begin{array}{c}
n_{2} \\
2
\end{array}\right)+18\left(\begin{array}{c}
n_{3} \\
2
\end{array}\right)+2 n_{1} n_{2}+6 n_{2} n_{3}+6 n_{3} n_{1}+n_{1}+2 n_{2}+9 n_{3}} .
\end{aligned}
$$

Our task in this section is to find a recurrence for $\tilde{a}(M)$. To do so, we require the Mathematica package qMultiSum implemented by Riese [18] of Research Institute for Symbolic Computation (RISC) of Johannes Kepler University. This package can be downloaded at the website of RISC:

https://www3.risc.jku.at/research/combinat/software/ergosum/index.html

We first import this package:

$$
<<\text { RISC'qMultisum' }
$$

Then the following recurrence can be computed automatically by calling the commands:

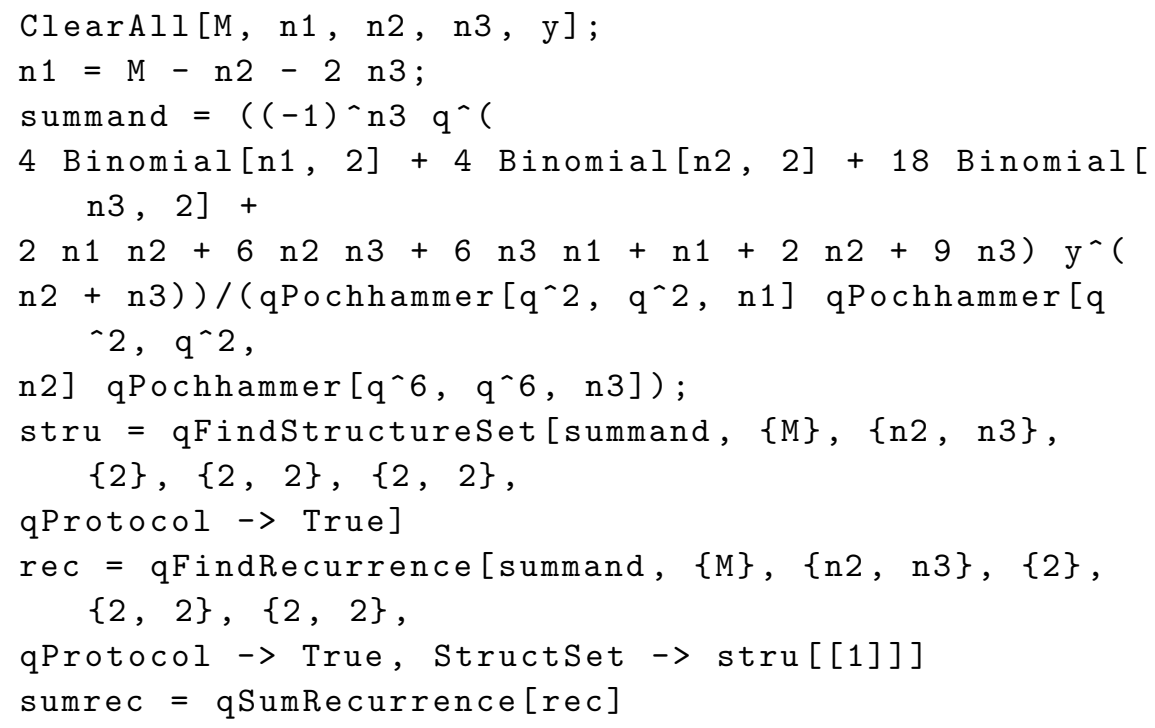

Theorem 5.1. For any $M \geq 0$,

$$
\begin{aligned}
0= & q^{12 M+24} y^{2}\left(1+2 y q+y^{2} q^{2}+q^{6 M+22}+y q^{6 M+23}+y^{2} q^{6 M+24}\right) \tilde{a}(M) \\
& -q^{12 M+27}(1+y q)\left(1+y q+y^{2} q^{2}-y^{2} q^{8}+y^{3} q^{3}+y^{4} q^{4}\right. \\
& \left.+q^{6 M+22}+y^{2} q^{6 M+24}+y^{4} q^{6 M+26}\right) \tilde{a}(M+1) \\
& -q^{6 M+17}\left(y+y q^{2}+2 y^{2} q+2 y^{2} q^{3}+y^{3} q^{2}+y^{3} q^{4}-q^{6 M+15}+q^{6 M+21}\right. \\
& -2 y q^{6 M+16}+2 y q^{6 M+22}+y q^{6 M+24}-y q^{12 M+38}
\end{aligned}
$$




$$
\begin{aligned}
& -3 y^{2} q^{6 M+17}+2 y^{2} q^{6 M+23}+y^{2} q^{6 M+25}-y^{2} q^{12 M+39} \\
& -2 y^{3} q^{6 M+18}+2 y^{3} q^{6 M+24}+y^{3} q^{6 M+26}-y^{3} q^{12 M+40} \\
& \left.-y^{4} q^{6 M+19}+y^{4} q^{6 M+25}\right) \tilde{a}(M+2) \\
- & q^{6 M+17}\left(1+q^{2}+q^{4}\right)(1+y q)\left(1+y q+y q^{3}+y^{2} q^{2}\right. \\
& \left.+q^{6 M+20}+y q^{6 M+21}+y^{2} q^{6 M+22}\right) \tilde{a}(M+3) \\
+ & \left(1-q^{6 M+24}\right)\left(1+2 y q+y^{2} q^{2}+q^{6 M+16}+y q^{6 M+17}+y^{2} q^{6 M+18}\right) \tilde{a}(M+4) .
\end{aligned}
$$

\section{Proof of (1.6)}

By (2.10), we find that to prove (1.6), it suffices to show that for $M \geq 0$,

$$
a(M)=\tilde{a}(M) .
$$

Recall from Corollary 3.2 and Theorem 5.1 that both $a(M)$ and $\tilde{a}(M)$ satisfy a recurrence. By a result of Kauers and Koutschan [16], we know that so does $a(M)-$ $\tilde{a}(M)$. To compute the recurrence for $a(M)-\tilde{a}(M)$, we require the Mathematica package qGeneratingFunctions implemented by Koutschan. This package can also

be downloaded at the website of RISC, the url of which is listed at the beginning of Section 5 .

To begin with, we import this package:

$<$ RISC 'qGeneratingFunctions '

Now assuming that sumrec1 $==0$ is the recurrence for $a(M)$ as in Corollary 3.2 and that sumrec $2==0$ is the recurrence for $\tilde{a}(M)$ as in Theorem 5.1, then the recurrence for $a(M)-\tilde{a}(M)$ can be computed by calling the commands:

\section{ClearAll $[\mathrm{M}, \mathrm{y}]$;}

QREPlus $[\{$ sumrec1 $==0\},\{$ sumrec $2==0\}$, SUM $[M]]$

The result gives us a fifth-order recurrence.

Theorem 6.1. Let $d(M):=a(M)-\tilde{a}(M)$. Then for $M \geq 5$,

$$
\begin{aligned}
q^{29}\left(1-q^{6 M}\right) d(M)= & -q^{12 M} y^{2}(1+y q) d(M-5) \\
+ & q^{12 M+3}\left(1+y q-y^{2} q^{8}+y^{3} q^{3}+y^{4} q^{4}\right) d(M-4) \\
+ & q^{6 M+9}(1+y q)\left(y q^{14}+y q^{16}\right. \\
& \left.+q^{6 M+5}-y q^{6 M}-y q^{6 M+6}+y^{2} q^{6 M+7}\right) d(M-3) \\
+ & q^{6 M+20}\left(q^{3}+q^{5}+q^{7}+y q^{2}+2 y q^{4}+2 y q^{6}\right. \\
& \left.+2 y q^{8}+y q^{10}+y^{2} q^{5}+y^{2} q^{7}+y^{2} q^{9}-y q^{6 M}\right) d(M-2) \\
- & q^{24}(1+y q)\left(q^{12}-q^{6 M}\right. \\
& \left.-q^{6 M+2}-q^{6 M+4}-q^{6 M+6}\right) d(M-1) .
\end{aligned}
$$

Therefore, to prove (6.1) for any $M \geq 0$, it suffices to verify that it holds true for $0 \leq M \leq 4$. This can be done by a straightforward calculation. 


\section{Proof of Corollary 1.2}

Taking $y=x$ in (1.6), we see that Theorem $\mathrm{A}$ is equivalent to (1.9):

$$
\begin{aligned}
\prod_{n \geq 0}\left(1+x q^{2 n+1}+x^{2} q^{4 n+2}\right) & =\sum_{\substack{n_{1}, n_{2}, n_{3} \geq 0 \\
\left(q^{2} ; q^{2}\right)_{n_{1}}\left(q^{2} ; q^{2}\right)_{n_{2}}\left(q^{6} ; q^{6}\right)_{n_{3}}}} \frac{(-1)^{n_{3}} x^{n_{1}+2 n_{2}+3 n_{3}}}{\left(\begin{array}{c}
n_{1} \\
2
\end{array}\right)+4\left(\begin{array}{c}
n_{2} \\
2
\end{array}\right)+18\left(\begin{array}{c}
n_{3} \\
2
\end{array}\right)+2 n_{1} n_{2}+6 n_{2} n_{3}+6 n_{3} n_{1}+n_{1}+2 n_{2}+9 n_{3}} .
\end{aligned}
$$

In this section, we give two proofs of this identity — one is $q$-hypergeometric and the other is computer-assisted.

For convenience, we write

$$
\begin{aligned}
S(x)=\sum_{M \geq 0} s(M) x^{M} & :=\sum_{n_{1}, n_{2}, n_{3} \geq 0} \frac{(-1)^{n_{3}} x^{n_{1}+2 n_{2}+3 n_{3}}}{\left(q^{2} ; q^{2}\right)_{n_{1}}\left(q^{2} ; q^{2}\right) n_{2}\left(q^{6} ; q^{6}\right) n_{3}} \\
& \times q^{4\left(\begin{array}{c}
n_{1} \\
2
\end{array}\right)+4\left(\begin{array}{c}
n_{2} \\
2
\end{array}\right)+18\left(\begin{array}{c}
n_{3} \\
2
\end{array}\right)+2 n_{1} n_{2}+6 n_{2} n_{3}+6 n_{3} n_{1}+n_{1}+2 n_{2}+9 n_{3}} .
\end{aligned}
$$

7.1. A $\boldsymbol{q}$-hypergeometric proof. For convenience, we write for integers $\beta_{1}, \beta_{2}$ and $\beta_{3}$ :

$$
\begin{aligned}
\sigma\left(\beta_{1}, \beta_{2}, \beta_{3}\right):= & \frac{(-1)^{n_{3}} x^{n_{1}+2 n_{2}+3 n_{3}}}{\left(q^{2} ; q^{2}\right)_{n_{1}}\left(q^{2} ; q^{2}\right)_{n_{2}}\left(q^{6} ; q^{6}\right)_{n_{3}}} \\
& \times q^{4\left(\begin{array}{c}
n_{1} \\
2
\end{array}\right)+4\left(\begin{array}{c}
n_{2} \\
2
\end{array}\right)+18\left(\begin{array}{c}
n_{3} \\
2
\end{array}\right)+2 n_{1} n_{2}+6 n_{2} n_{3}+6 n_{3} n_{1}+\beta_{1} n_{1}+\beta_{2} n_{2}+\beta_{3} n_{3}}
\end{aligned}
$$

and

$$
\Sigma\left(\beta_{1}, \beta_{2}, \beta_{3}\right):=\sum_{n_{1}, n_{2}, n_{3} \geq 0} \sigma\left(\beta_{1}, \beta_{2}, \beta_{3}\right) .
$$

Our proof relies on the following lemma.

Lemma 7.1. Let $k_{1}, k_{2}$ and $k_{3}$ be nonnegative integers. Then for any integers $\beta_{1}$, $\beta_{2}$ and $\beta_{3}$,

$$
\begin{aligned}
& \sum_{n_{1}, n_{2}, n_{3} \geq 0} \sigma\left(\beta_{1}, \beta_{2}, \beta_{3}\right)\left(q^{2 n_{1}} ; q^{-2}\right)_{k_{1}}\left(q^{2 n_{2}} ; q^{-2}\right)_{k_{2}}\left(q^{6 n_{3}} ; q^{-6}\right)_{k_{3}} \\
= & (-1)^{k_{3}} x^{k_{1}+2 k_{2}+3 k_{3}} q^{4}\left(\begin{array}{c}
k_{1} \\
2
\end{array}\right)+4\left(\begin{array}{c}
k_{2} \\
2
\end{array}\right)+18\left(\begin{array}{c}
k_{3} \\
2
\end{array}\right)+2 k_{1} k_{2}+6 k_{2} k_{3}+6 k_{3} k_{1}+k_{1} \beta_{1}+k_{2} \beta_{2}+k_{3} \beta_{3} \\
& \times \Sigma\left(\beta_{1}+4 k_{1}+2 k_{2}+6 k_{3}, \beta_{2}+2 k_{1}+4 k_{2}+6 k_{3}, \beta_{3}+6 k_{1}+6 k_{2}+18 k_{3}\right) .
\end{aligned}
$$

Proof. We have

$$
\begin{aligned}
& \sum_{n_{1}, n_{2}, n_{3} \geq 0} \sigma\left(\beta_{1}, \beta_{2}, \beta_{3}\right)\left(q^{2 n_{1}} ; q^{-2}\right)_{k_{1}}\left(q^{2 n_{2}} ; q^{-2}\right)_{k_{2}}\left(q^{6 n_{3}} ; q^{-6}\right)_{k_{3}} \\
= & \sum_{n_{1}, n_{2}, n_{3} \geq 0} \frac{(-1)^{n_{3}} x^{n_{1}+2 n_{2}+3 n_{3}}\left(q^{2 n_{1}} ; q^{-2}\right)_{k_{1}}\left(q^{2 n_{2}} ; q^{-2}\right)_{k_{2}}\left(q^{6 n_{3}} ; q^{-6}\right)_{k_{3}}}{\left(q^{2} ; q^{2}\right)_{n_{1}}\left(q^{2} ; q^{2}\right)_{n_{2}}\left(q^{6} ; q^{6}\right)_{n_{3}}} \\
& \times q^{4\left(\begin{array}{c}
n_{1} \\
2
\end{array}\right)+4\left(\begin{array}{c}
n_{2} \\
2
\end{array}\right)+18\left(\begin{array}{c}
n_{3} \\
2
\end{array}\right)+2 n_{1} n_{2}+6 n_{2} n_{3}+6 n_{3} n_{1}+\beta_{1} n_{1}+\beta_{2} n_{2}+\beta_{3} n_{3}} \\
= & \sum_{\substack{n_{1} \geq k_{1} \\
n_{2} \geq k_{2} \\
n_{3} \geq k_{3}}} \frac{(-1)^{n_{3}} x^{n_{1}+2 n_{2}+3 n_{3}}}{\left(q^{2} ; q^{2}\right)_{n_{1}-k_{1}}\left(q^{2} ; q^{2}\right)_{n_{2}-k_{2}}\left(q^{6} ; q^{6}\right)_{n_{3}-k_{3}}} \\
& \times q^{4\left(\begin{array}{c}
n_{1} \\
2
\end{array}\right)+4\left(\begin{array}{c}
n_{2} \\
2
\end{array}\right)+18\left(\begin{array}{c}
n_{3} \\
2
\end{array}\right)+2 n_{1} n_{2}+6 n_{2} n_{3}+6 n_{3} n_{1}+\beta_{1} n_{1}+\beta_{2} n_{2}+\beta_{3} n_{3}}
\end{aligned}
$$




$$
\begin{aligned}
& =\sum_{n_{1}, n_{2}, n_{3} \geq 0} \frac{(-1)^{n_{3}+k_{3}} x^{\left(n_{1}+k_{1}\right)+2\left(n_{2}+k_{2}\right)+3\left(n_{3}+k_{3}\right)}}{\left(q^{2} ; q^{2}\right)_{n_{1}}\left(q^{2} ; q^{2}\right)_{n_{2}}\left(q^{6} ; q^{6}\right)_{n_{3}}} \\
& \quad \times q^{4\left(\begin{array}{c}
n_{1}+k_{1} \\
n_{2}
\end{array}\right)+4\left(\begin{array}{c}
n_{2}+k_{2} \\
2
\end{array}\right)+18\left({ }_{2}{ }_{2}^{+k_{3}}\right)+2\left(n_{1}+k_{1}\right)\left(n_{2}+k_{2}\right)+6\left(n_{2}+k_{2}\right)\left(n_{3}+k_{3}\right)+6\left(n_{3}+k_{3}\right)\left(n_{1}+k_{1}\right)} \\
& \quad \times q^{\beta_{1}\left(n_{1}+k_{1}\right)+\beta_{2}\left(n_{2}+k_{2}\right)+\beta_{3}\left(n_{3}+k_{3}\right)} .
\end{aligned}
$$

This gives (7.2) after simplification.

Now, we show the following result.

Theorem 7.2. We have

$$
\Sigma(1,2,9)=\left(1+x q+x^{2} q^{2}\right) \Sigma(3,6,15) .
$$

Proof. We split 1 as

$$
1=I_{1}+I_{2}+I_{3}+I_{4}+I_{5}+I_{6}+I_{7}+I_{8}+I_{9},
$$

where

$$
\begin{aligned}
& I_{1}=q^{2 n_{1}+4 n_{2}+6 n_{3}} \\
& I_{2}=q^{-2 n_{1}+2 n_{2}+2}\left(1-q^{2 n_{1}}\right), \\
& I_{3}=1-q^{2 n_{2}} \\
& I_{4}=q^{4 n_{1}+2 n_{2}+6 n_{3}}\left(1-q^{2 n_{2}}\right) \\
& I_{5}=-q^{-2 n_{1}+2 n_{2}+2}\left(1-q^{2 n_{1}}\right)\left(1-q^{2 n_{1}-2}\right), \\
& I_{6}=q^{2 n_{1}+4 n_{2}+6 n_{3}-2}\left(1-q^{2 n_{1}}\right)\left(1-q^{2 n_{2}}\right), \\
& I_{7}=q^{2 n_{1}+4 n_{2}}\left(1-q^{6 n_{3}}\right) \\
& I_{8}=q^{2 n_{1}+2 n_{2}+6 n_{3}}\left(1-q^{2 n_{1}}\right)\left(1-q^{2 n_{2}}\right)\left(1-q^{2 n_{2}-2}\right), \\
& I_{9}=q^{2 n_{1}+2 n_{2}}\left(1-q^{2 n_{2}}\right)\left(1-q^{6 n_{3}}\right) .
\end{aligned}
$$

Then by Lemma 7.1,

$$
\begin{aligned}
\sum_{n_{1}, n_{2}, n_{3} \geq 0} \sigma(1,2,9) I_{1} & =\sum_{n_{1}, n_{2}, n_{3} \geq 0} \sigma(3,6,15) \\
& =\Sigma(3,6,15), \\
\sum_{n_{1}, n_{2}, n_{3} \geq 0} \sigma(1,2,9) I_{2} & =q^{2} \sum_{n_{1}, n_{2}, n_{3} \geq 0} \sigma(-1,4,9)\left(1-q^{2 n_{1}}\right) \\
& =x q \Sigma(3,6,15), \\
\sum_{n_{1}, n_{2}, n_{3} \geq 0} \sigma(1,2,9) I_{3} & =\sum_{n_{1}, n_{2}, n_{3} \geq 0} \sigma(1,2,9)\left(1-q^{2 n_{2}}\right) \\
& =x^{2} q^{2} \Sigma(3,6,15), \\
\sum_{n_{1}, n_{2}, n_{3} \geq 0} \sigma(1,2,9) I_{4} & =\sum_{n_{1}, n_{2}, n_{3} \geq 0} \sigma(5,4,15)\left(1-q^{2 n_{2}}\right) \\
& =x^{2} q^{4} \Sigma(7,8,21), \\
\sum_{n_{1}, n_{2}, n_{3} \geq 0} \sigma(1,2,9) I_{5} & =-q^{2} \sum_{n_{1}, n_{2}, n_{3} \geq 0} \sigma(-1,4,9)\left(1-q^{2 n_{1}}\right)\left(1-q^{2 n_{1}-2}\right) \\
& =-x^{2} q^{4} \Sigma(7,8,21),
\end{aligned}
$$




$$
\begin{aligned}
\sum_{n_{1}, n_{2}, n_{3} \geq 0} \sigma(1,2,9) I_{6} & =q^{-2} \sum_{n_{1}, n_{2}, n_{3} \geq 0} \sigma(3,6,15)\left(1-q^{2 n_{1}}\right)\left(1-q^{2 n_{2}}\right) \\
& =x^{3} q^{9} \Sigma(9,12,27), \\
\sum_{n_{1}, n_{2}, n_{3} \geq 0} \sigma(1,2,9) I_{7} & =\sum_{n_{1}, n_{2}, n_{3} \geq 0} \sigma(3,6,9)\left(1-q^{6 n_{3}}\right) \\
& =-x^{3} q^{9} \Sigma(9,12,27), \\
\sum_{n_{1}, n_{2}, n_{3} \geq 0} \sigma(1,2,9) I_{8} & =\sum_{n_{1}, n_{2}, n_{3} \geq 0} \sigma(3,4,15)\left(1-q^{2 n_{1}}\right)\left(1-q^{2 n_{2}}\right)\left(1-q^{2 n_{2}-2}\right) \\
& =x^{5} q^{19} \Sigma(11,14,33), \\
\sum_{n_{1}, n_{2}, n_{3} \geq 0} \sigma(1,2,9) I_{9} & =\sum_{n_{1}, n_{2}, n_{3} \geq 0} \sigma(3,4,9)\left(1-q^{6 n_{3}}\right) \\
& =-x^{5} q^{19} \Sigma(11,14,33) .
\end{aligned}
$$

Therefore,

$$
\begin{aligned}
\Sigma(1,2,9) & =\sum_{n_{1}, n_{2}, n_{3} \geq 0} \sigma(1,2,9)\left(I_{1}+I_{2}+I_{3}+I_{4}+I_{5}+I_{6}+I_{7}+I_{8}+I_{9}\right) \\
& =\Sigma(3,6,15)+x q \Sigma(3,6,15)+x^{2} q^{2} \Sigma(3,6,15)
\end{aligned}
$$

which is our desired result.

First proof of (1.9). From (7.1), we have $S(x)=\Sigma(1,2,9)$ and $S\left(x q^{2}\right)=\Sigma(3,6,15)$. It follows from Theorem 7.2 that

$$
S(x)=\left(1+x q+x^{2} q^{2}\right) S\left(x q^{2}\right) .
$$

Finally, we conclude by $S(0)=1$ that

$$
S(x)=\prod_{n \geq 0}\left(1+x q^{2 n+1}+x^{2} q^{4 n+2}\right),
$$

which is (1.9).

7.2. A computer-assisted proof. Here, our task is to derive a recurrence for the coefficients $s(M)$ in (7.1), which is also computed by the qMultiSum package. We call the following commands:

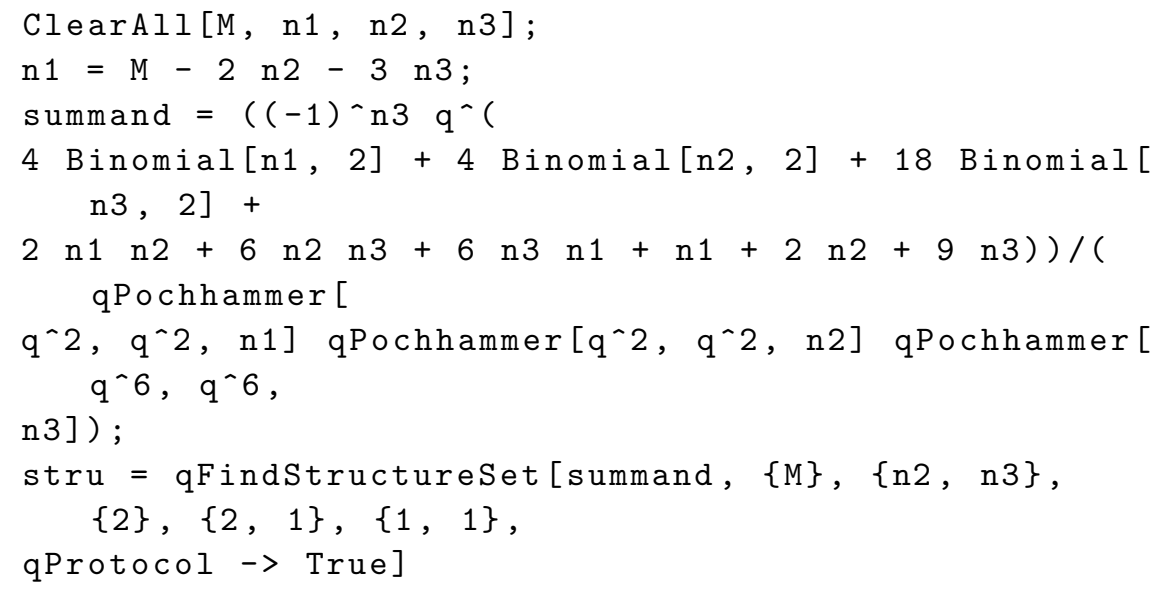


rec $=$ qFindRecurrence [summand, $\{M\},\{n 2, n 3\},\{2\}$,

$\{2,1\},\{1,1\}$,

qProtocol $\rightarrow$ True, Structset $\rightarrow$ stru[[1]]]

sumrec $=$ qSumRecurrence $[r e c]$

Then we arrive at a sixth-order recurrence.

Theorem 7.3. For $M \geq 0$,

$$
\begin{aligned}
0= & q^{6 M+24} s(M)-q^{4 M+18}\left(1+q^{2}+q^{4}\right) s(M+2)-q^{6 M+27} s(M+3) \\
& +q^{2 M+10}\left(1+q^{2}+q^{4}-q^{2 M+12}\right) s(M+4)+q^{4 M+21} s(M+5) \\
& -\left(1-q^{2 M+12}\right) s(M+6) .
\end{aligned}
$$

Now, we translate this recurrence to a $q$-difference equation for $S(x)$.

Corollary 7.4. We have

$$
\begin{aligned}
0= & S(x)-\left[x^{2} q^{2}\left(1+q^{2}+q^{4}\right)+1\right] S\left(x q^{2}\right) \\
& +\left[x^{4} q^{10}\left(1+q^{2}+q^{4}\right)+x^{2} q^{6}-x q\right] S\left(x q^{4}\right)-\left[x^{6} q^{24}-x^{3} q^{9}\right] S\left(x q^{6}\right) .
\end{aligned}
$$

Proof. We multiply by $x^{M+6}$ on both sides of the recurrence in Theorem 7.3, and sum over $M \geq 0$. Then

$$
\begin{aligned}
0= & x^{6} q^{24} S\left(x q^{6}\right)-x^{4} q^{10}\left(1+q^{2}+q^{4}\right)\left(S\left(x q^{4}\right)-\sum_{k=0}^{1} s(k)\left(x q^{4}\right)^{k}\right) \\
& -x^{3} q^{9}\left(S\left(x q^{6}\right)-\sum_{k=0}^{2} s(k)\left(x q^{6}\right)^{k}\right)+x^{2} q^{2}\left(1+q^{2}+q^{4}\right)\left(S\left(x q^{2}\right)-\sum_{k=0}^{3} s(k)\left(x q^{2}\right)^{k}\right) \\
& -x^{2} q^{6}\left(S\left(x q^{4}\right)-\sum_{k=0}^{3} s(k)\left(x q^{4}\right)^{k}\right)+x q\left(S\left(x q^{4}\right)-\sum_{k=0}^{4} s(k)\left(x q^{4}\right)^{k}\right) \\
& -\left(S(x)-\sum_{k=0}^{5} s(k) x^{k}\right)+\left(S\left(x q^{2}\right)-\sum_{k=0}^{5} s(k)\left(x q^{2}\right)^{k}\right) .
\end{aligned}
$$

Inserting the values of $s(0), \ldots, s(5)$ yields the desired result.

Second proof of (1.9). Notice that if any $F(x) \in \mathbb{C}[[q]][[x]]$ satisfies the $q$-difference equation in Corollary 7.4, then it is uniquely determined by $F(0)$. Therefore, if we write

$$
P(x):=\prod_{n \geq 0}\left(1+x q^{2 n+1}+x^{2} q^{4 n+2}\right),
$$

by noticing that $S(0)=P(0)=1$, it suffices to verify that

$$
\begin{aligned}
0= & P(x)-\left[x^{2} q^{2}\left(1+q^{2}+q^{4}\right)+1\right] P\left(x q^{2}\right) \\
& +\left[x^{4} q^{10}\left(1+q^{2}+q^{4}\right)+x^{2} q^{6}-x q\right] P\left(x q^{4}\right)-\left[x^{6} q^{24}-x^{3} q^{9}\right] P\left(x q^{6}\right) .
\end{aligned}
$$

Namely,

$$
\begin{aligned}
0= & \left(1+x q+x^{2} q^{2}\right)\left(1+x q^{3}+x^{2} q^{6}\right)\left(1+x q^{5}+x^{2} q^{10}\right) \\
& -\left[x^{2} q^{2}\left(1+q^{2}+q^{4}\right)+1\right]\left(1+x q^{3}+x^{2} q^{6}\right)\left(1+x q^{5}+x^{2} q^{10}\right) \\
& +\left[x^{4} q^{10}\left(1+q^{2}+q^{4}\right)+x^{2} q^{6}-x q\right]\left(1+x q^{5}+x^{2} q^{10}\right) \\
& -\left[x^{6} q^{24}-x^{3} q^{9}\right] .
\end{aligned}
$$


This can be checked easily.

Thus, we have $P(x)=S(x)$, which is exactly (1.9).

\section{Conclusion}

In light of the fact that Schur's theorem seems to be at the center of the theory of partition identities (perhaps even more so than the Rogers-Ramanujan identities), it is a major step forward to have this theorem now embedded in the theory of linked partition ideals. The point of paper [8] was essentially to make the case that Alladi's addition to Schur's theorem is actually the core of this result, and identity (1.9) certainly reaffirms that observation.

It is clear that the theory of linked partition ideals is still in its infancy after almost forty years. The goal would be a classification theorem for linked partition ideals comparable to the classification theorem [6, Theorem 8.4, p. 126] for partition ideals of order 1 . With the advent of powerful computer algebra packages, we have seen advances such as obtained in this paper and in [12] and [13]. We hope to see much progress in the future.

Acknowledgements. The first author was supported by a grant (\#633284) from the Simons Foundation. The second author was supported by a Killam Postdoctoral Fellowship from the Killam Trusts.

\section{References}

1. K. Alladi and B. Gordon, Schur's partition theorem, companions, refinements and generalizations, Trans. Amer. Math. Soc. 347 (1995), no. 5, 1591-1608.

2. G. E. Andrews, Partition identities, Advances in Math. 9 (1972), 10-51.

3. G. E. Andrews, An analytic generalization of the Rogers-Ramanujan identities for odd moduli, Proc. Nat. Acad. Sci. U.S.A. 71 (1974), 4082-4085.

4. G. E. Andrews, A general theory of identities of the Rogers-Ramanujan type, Bull. Amer. Math. Soc. 80 (1974), 1033-1052.

5. G. E. Andrews, Problems and prospects for basic hypergeometric functions, In: Theory and application of special functions (Proc. Advanced Sem., Math. Res. Center, Univ. Wisconsin, Madison, Wis., 1975), 191-224, Math. Res. Center, Univ. Wisconsin, Publ. No. 35, Academic Press, New York, 1975.

6. G. E. Andrews, The theory of partitions, Reprint of the 1976 original. Cambridge Mathematical Library. Cambridge University Press, Cambridge, 1998. xvi+255 pp.

7. G. E. Andrews, Schur's theorem, partitions with odd parts and the Al-Salam-Carlitz polynomials, In: q-Series from a contemporary perspective (South Hadley, MA, 1998), 45-56, Contemp. Math., 254, Amer. Math. Soc., Providence, RI, 2000.

8. G. E. Andrews, The Alladi-Schur polynomials and their factorization, In: Analytic number theory, modular forms and q-hypergeometric series, 25-38, Springer Proc. Math. Stat., 221, Springer, Cham, 2017.

9. G. E. Andrews, A refinement of the Alladi-Schur theorem, In: Lattice path combinatorics and applications, 71-77, Dev. Math., 58, Springer, Cham, 2019.

10. G. E. Andrews, K. Bringmann, and K. Mahlburg, Double series representations for Schur's partition function and related identities, J. Combin. Theory Ser. A 132 (2015), 102-119.

11. S. Chern, Linked partition ideals, directed graphs and $q$-multi-summations, Electron. J. Combin. 27 (2020), no. 3, Paper No. 3.33, 29 pp.

12. S. Chern, Linked partition ideals and Andrews-Gordon type series for Alladi and Gordon's extension of Schur's identity, submitted.

13. S. Chern and Z. Li, Linked partition ideals and Kanade-Russell conjectures, Discrete Math. 343 (2020), no. 7, 111876, 24 pp.

14. W. Gleißberg, Über einen Satz von Herrn I. Schur, Math. Z. 28 (1928), no. 1, 372-382. 
15. B. Gordon, A combinatorial generalization of the Rogers-Ramanujan identities, Amer. J. Math. 83 (1961), 393-399.

16. M. Kauers and C. Koutschan, A Mathematica package for $q$-holonomic sequences and power series, Ramanujan J. 19 (2009), no. 2, 137-150.

17. K. Kurşungöz, Andrews-Gordon type series for Schur's partition identity, Discrete Math. 344 (2021), no. 11, Paper No. 112563, 31 pp.

18. A. Riese, qMultiSum - a package for proving $q$-hypergeometric multiple summation identities, J. Symbolic Comput. 35 (2003), no. 3, 349-376.

19. I. Schur, Zur additiven Zahlentheorie, S.-B. Preuss. Akad. Wiss. Phys.-Math. Kl. (1926), 488-495.

(G. E. Andrews) Department of Mathematics, The Pennsylvania State University, UniVERSITY PARK, PA 16802, USA

Email address: gea1@psu.edu

(S. Chern) Department of Mathematics and Statistics, Dalhousie University, Halifax, Nova Scotia, B3H 4R2, CANAdA

Email address: chenxiaohang92@gmail.com

(Z. Li) Department of Mathematics, The Pennsylvania State University, University PARK, PA 16802, USA

Email address: zf15082@psu.edu 